\title{
Re-commissioning of the VLT/UT4 telescope after the Deformable Secondary Mirror installation
}

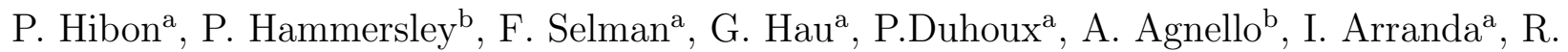

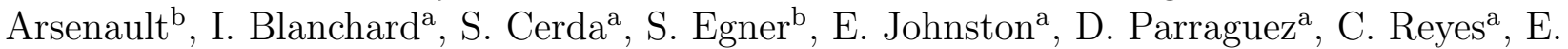 \\ Vernet $^{\mathrm{b}}$, AOF Team ${ }^{\mathrm{b}}$, UT4 Team ${ }^{\mathrm{a}}$, and VLTI Team ${ }^{\mathrm{a}}$ \\ ${ }^{a}$ ESO Chile, Alonso de Cordova 3107, Santiago, Chile \\ ${ }^{\mathrm{b}} \mathrm{ESO}$, Address, Garching, Germany
}

\begin{abstract}
During the 2nd semester of 2016, a new Deformable Secondary Mirror (DSM), part of the Adaptive Optics Facility Project at Paranal Observatory, was installed on the Unit Telescope 4. Starting end of November 2016, we then re-commissioned the telescope and the following three instruments : HAWK-I, MUSE and SINFONI. It is important to understand how a DSM, even if used in non-adaptive optics mode (i.e., in its rest/flat position), can impact the operations and the quality of the observations. We discuss here the results of this telescope and instrument re-commissioning, the challenges and problems we met, and compare the new performance to the ones obtained previously with the traditional Dornier Secondary Mirror.
\end{abstract}

Keywords: Deformable Mirror, Adaptive Optics, commissioning, VLT

\section{TELESCOPE}

\subsection{The Deformable Secondary Mirror}

The AOF DSM is featuring an optical shell of $1.1 \mathrm{~m}$ diameter and $2 \mathrm{~mm}$ thin, floating on a magnetic field created by 1170 voice coil actuators (Ref. 1,2). When in non-AO mode, the DSM optical shape is set to its best flat, with a surface figure standard deviation as small as $10 \mathrm{~nm}$ rms compared to the theoretical optical prescription of the telescope. Additionally, an hexapod is used to control the rigid-body motion of the DSM in the telescope coordinate system, by adjusting the longitudinal position of its vertex along the telescope optical axis (focus control) and its lateral position (pupil lateral motion). More details on the telescope active optics control are given in (Ref. 3). During the re-commissioning of the UT4 and its instruments, the DSM was only used in non-AO mode.

The orientation of the movements of the DSM with respect to the rest of the telescope is important, as the hexapod is requested to move in operation as part of the active optics system. This movement must be both of the correct size and in the correct direction. The test that was made was 1) Take two images with HAWK-I offset in declination by 60 arc seconds (on sky) 2) Tilt the DSM hexapod by 60 arc seconds (mechanical angle) in elevation and take an image on HAWK-I The distance moved by the two offsets can then be compared. The angle between the two movements should be the same as the parallactic angle. This was repeated 6 times on the sky. The DSM was able to correctly focus for the three focus position (Cassegrain, Nasmyth A and Nasmyth B.) with more than sufficient range for the expected active optics corrections. The active optics would correct significant focus errors (focus blurring of a few arc seconds) and reach a stable value within two active optics cycles.

Further author information: (Send correspondence to P. Hibon)

P. Hibon.: E-mail: phibon@eso.org 


\subsection{Image Quality}

Directly measuring image quality is very difficult as it is dependent on seeing, atmospheric refraction etc. Images were obtained in good seeing $(\approx 0.5)$, with the Dornier and the DSM. A qualitative comparison is then possible. A series of 10 second J-band images were taken of the Globular cluster M30 using the Dornier M2 and the DSM with HAWK-I. The important thing to note is that the PSFs obtained on these images are well defined and axisymmetric (see Section 3).

A test was made with both the Dornier M2 and the DSM pointing to various elevations, closing the active optics loop and then measuring the errors in focus, astigmatism, coma and trefoil on the DSM. Where possible, guide stars relatively close to the axis were chosen. As can be seen there is little difference between the two secondary mirrors, at least for elevations above 40degrees.

Ideally the image quality should be independent of the position of the guide star chosen. However, a misalignment of M1 w.r.t. M2 will lead to a change in the astigmatism and a tilt in the focal plane. In order to explore this, a test was made where a bright star was centered on the Maintenance and Commissioning Mode (MCM) (Ref. 4), various guide stars were chosen and the aberrations on the MCM measured after two cycles of the active optics on each guide star. The guide stars were up to 10 arc-minutes off axis and uniformly distributed across the focal plane. The measured errors on the MCM were then plotted against altitude/azimuth offset of the guide star from the coordinates of the centre of the field. (The MCM is a natural guide star 40x40 Shack-Hartmann wavefront sensor which can operate at 1000 frames per second. The projected size of each lenslet is about $2.7 \mathrm{~cm}$ on M2, The MCM is part of GRAAL. Unfortunately, the MCM introduces a small amount of astigmatism into the WFS so it is impossible to use this to absolutely determine the image quality.)

\subsection{Field Stabilization}

The Non AO operation of the DSM is in general comparable with the Dornier M2 Thermal background and Seeing limited image quality are comparable. Field stabilization (FS) proved to work from 8-65 frames per seconds. We also tested the field stabilization in windy conditions. In these conditions, the DSM is more sensitive to wind shake than the traditional Dornier mirror. The FS performance has drastically improved since the FS loop frequency was raised up to 65 fps. By deriving a new DSM flat, the high spatial frequency noise has been improved although some high order manufacturing aberrations are imprinted into the DSM flat.

\section{VLTI}

The VLT offers also the possibility of combining coherently the light from the four UTs to work as an interferometer. The Very Large Telescope Interferometer (VLTI), with its own suite of instruments, ultimately providing imagery at the milli arcsecond level as well as astrometry at 10 micro arcsecond precision.

We re-commissioned the VLTI after the installation of the DSM in UT4 in stand-alone operation during the night of the 16th November, and with UT1-2-3-4 during the two nights of 22nd and 23rd November 2016. Two out of the three VLTI instruments were available during this period : PIONIER and GRAVITY.

\subsection{Point Spread Function}

The shape of the PSF and the image quality with the correction by the VLTI adaptive optic system (MACAO) was measured on IRIS during a period with very good seeing (around $0.45 \operatorname{arcsec}$ ). For calculating the Strehlratio, 450 images in K-band were co-added, with an exposure time of 0.067s each (total integration time: 30.15 sec). From the images of the co-added PSF (see Figure 1), it is apparent that the residual wavefront aberrations are significantly higher on UT4 than on the other three UTs. The Strehl seems to be lower and there are more quasi-static speckles in the image. Due to high-spatial-frequency aberrations in the used flat pattern of the DSM, there was a significant speckle halo present in the images on the Infrared Image Sensor (IRIS). The flat pattern of the DSM was corrected which improved the image quality on IRIS. 

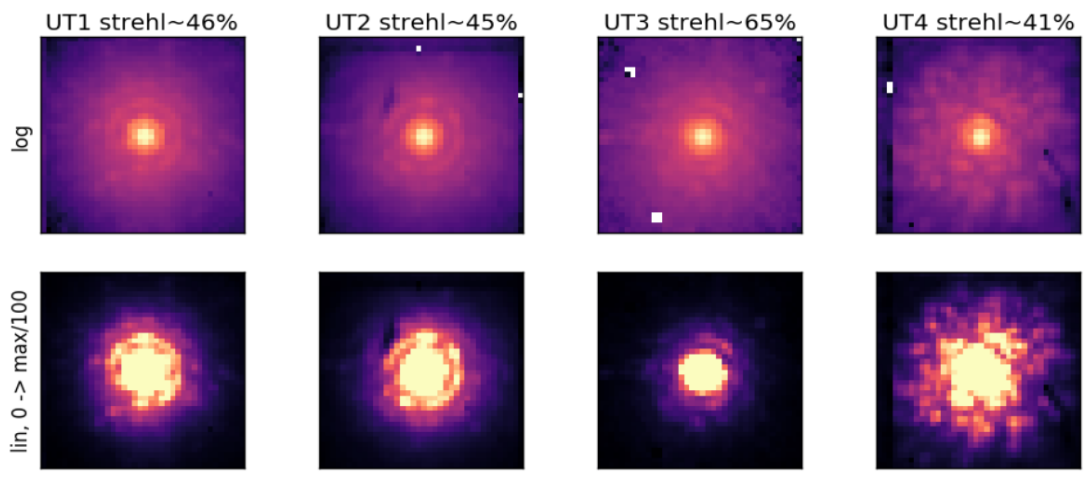

Figure 1. Comparison of the K-band PSF on IRIS for the 4 UTs with MACAO correction. Upper plots are in log scale, whereas the lower plots show the bottom $1 \%$ of the flux from the peak. The Strehl is computed as the ratio between the peak of the PSF to the peak the diffraction PSF of same total flux. This is not a very reliable method in the case of IRIS, since the PSF core is poorly sampled.

\subsection{PIONIER}

PIONIER is a H-band 4-beam combiner instrument at the VLT interferometer. It provides visibilities of six different baselines, as well as four closure phase measurements, simultaneously. PIONIER features low resolution spectroscopic optics to measure at six different wavelengths within the H-band, or can work in integrated light for sensitivity enhancement on faint targets.

The instrument contrast for PIONIER was good in all baselines. No difference to previous observations was found.

\subsection{GRAVITY}

GRAVITY is an interferometric instrument operating in the $\mathrm{K}$ band, between 2.0 and 2.4 microns. It combines 4 telescope beams and is designed to perform both interferometric imaging and astrometry by phase referencing. The instrument delivers spectrally dispersed interferometric quantities in low, medium, and high spectral resolution.

The Power Spectrum Density (PSD) of the residual optical path difference, as measured on 22nd November 2016 is shown in Figure 2 right. For comparison, a similar plot from the observations in September 2016 is shown in Figure 2 left. The amplitude of the vibrations is still an issue for interferometry. However, with the installation of the DSM, there were no new vibrations added to UT4.

After the installation of the DSM, most of the VLTI performance requirements of UT4 are still met. The only non-compliance, which related to the DSM is the quality of the PSF.

\section{HAWK-I}

HAWK-I is a cryogenic wide-field imager installed at the Nasmyth A focus of UT4 (Ref. 5-8). The on-sky field of view is 7.5'x7.5', with a cross-shaped gap of 15" between the four HAWAII 2RG 2048x2048 pixels detectors and a pixel scale of $0.106 "$.

The standard calibrations (dark level, non-linearity, for examples) were verified and do not show any change with the DSM installation.

\subsection{Photometry}

The Zero-Point magnitude in each broad-band filter was verified and are all very close to the normal average value. 

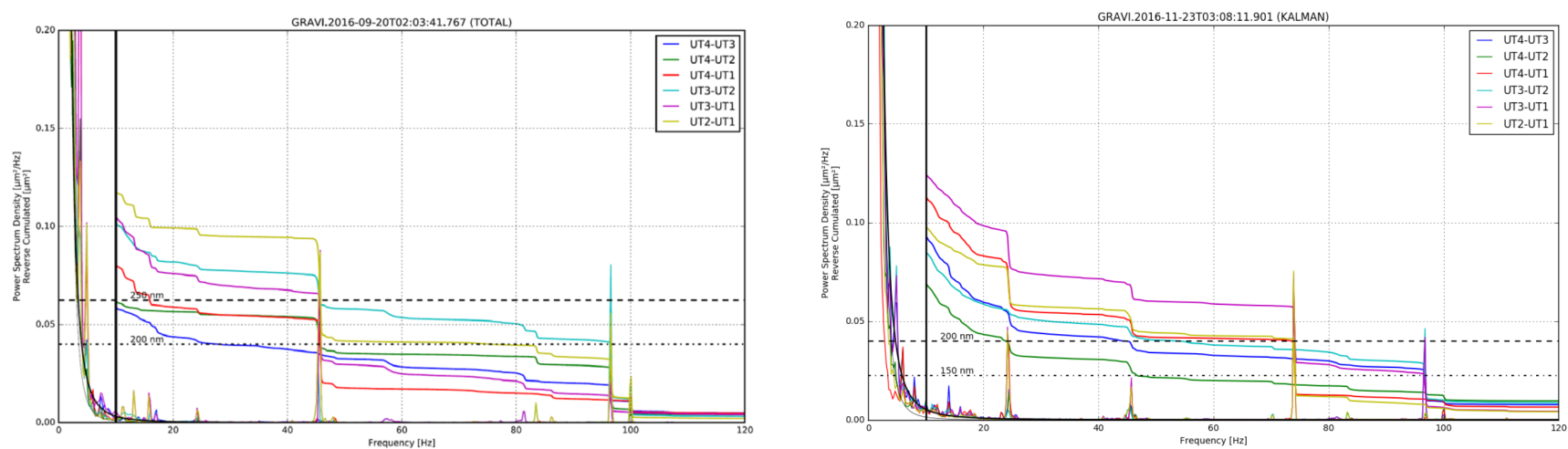

Figure 2. Left : The PSD of the vibration background at the UTs, as measured in September 2016, before the installation of the DSM. Right: The PSD of the vibration background at the UTs, as measured in November 2016, after the installation of the DSM.

\subsection{Distorsion}

No distortion was introduced in $\mathrm{Y}$ and $\mathrm{H}$ bands at 4\%. J-band shows less distortion in Nov than Sept.
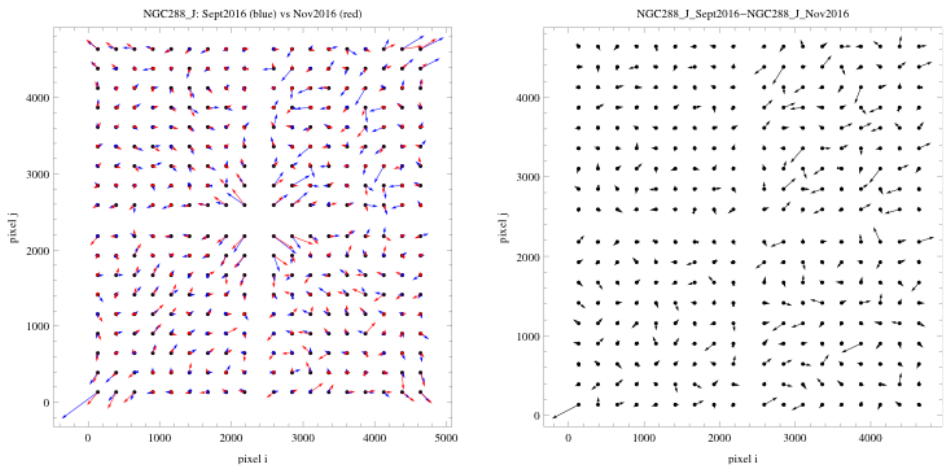

Figure 3. Distorsion maps done on NGC288 in J-band filters. Left : Distorsion maps showing the distorsion in September 2016 (before the DSM instalation) in red and in November 2016 in blue (after the DSM instalation). Right : Distorsion map showing the difference between the September and the November 2016 ones. We can remark on the right figure that the difference of distorsion possibly introduced by the DSM is negligible.

\subsection{FWHM}

We compared the FWHM across the whole field of view on a photometric field observed before and after the DSM installation, and looked at the ellipticity of the objects. Figures 4 and 5 show a comparison of the image quality in the different broad bands and the FWHM maps produced in a standard field. The ellipticity of the stars was also verified. We also took advantage of this re-commissioning to test the new HAWK-I pipeline. The difference in the average value of the FWHM is mostly due to the change of the ambient conditions between the pre- and post-commissioning tests.

\section{SINFONI}

SINFONI is a near-infrared $(1.1-2.45 \mathrm{~m})$ integral field spectrograph fed by an adaptive optics module, currently installed at the Cassegrain focus of UT4 (Ref. 9). The spectrograph operates with 4 gratings $(\mathrm{J}, \mathrm{H}, \mathrm{K}, \mathrm{H}+\mathrm{K})$, providing a spectral resolution around 2000, 3000, 4000 in $\mathrm{J}, \mathrm{H} \& \mathrm{~K}$ respectively, and 1500 in $\mathrm{H}+\mathrm{K}$. 


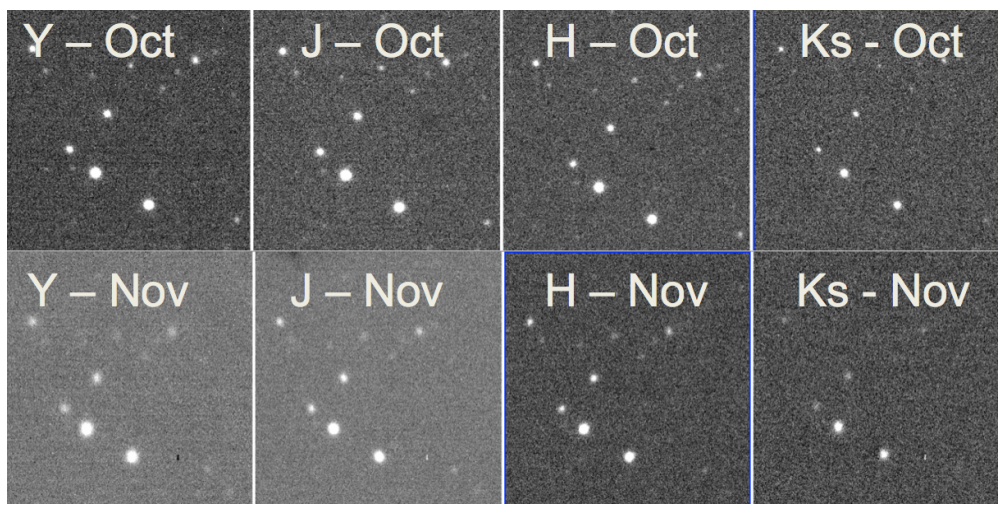

Figure 4. Illustration of the Image Quality obtained before and after the DSM installation in each broad-band filters.
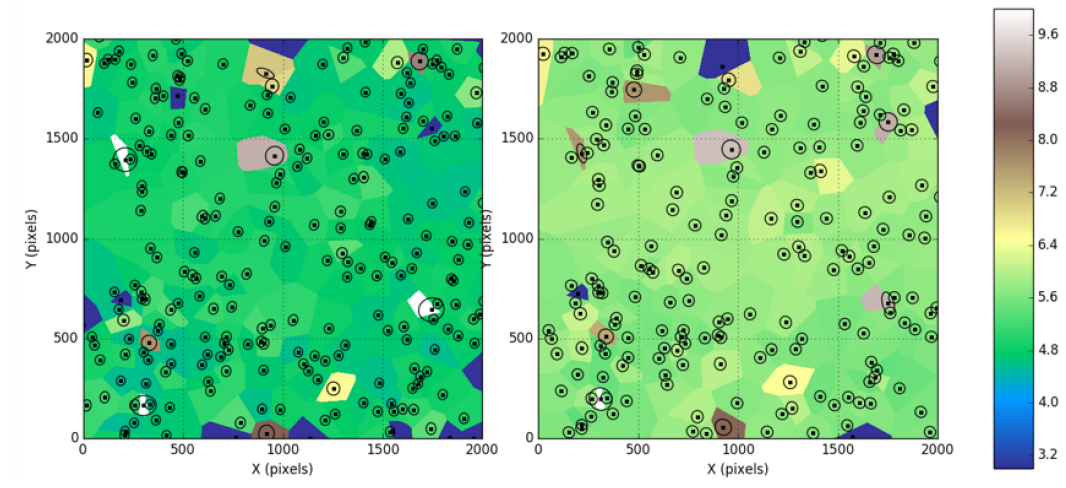

Figure 5. FWHM map for NGC 288 in H-band at airmass 1. The color scale represents the distribution of the FWHM values. The black circles show the ellipticity of the objects in the field.

All tested instrument functions, Dark, Strehl, Flat, Resolving power, Distortion map, Vignetting, Linearity and Gain, were tested and behave as they should, including integration and communication with other UT4 systems. All NGS, noAO, LGS and LGS-noTTS modes perform as expected. The NGS performance on a bright star achieves Strehl ratios as expected, especially in the redder bands such as K and H+K. A new flat for the DSM was implemented to eliminate the effects of the high-order aberrations introduced by the DSM to the PSF, especially for J. Figure 6 shows the comparison between the PSF taken in J and K-bands with the Dornier M2 and with the DSM M2 units.

\section{MUSE}

MUSE, the Multi-Unit Spectroscopic Explorer, is an Integral Field Spectrograph located at the Nasmyth B focus of Yepun, the VLT UT4 telescope (Ref. 10). It has a modular structure composed of 24 identical IFU modules that together sample, in Wide Field Mode (WFM), a near-contiguous 1 squared arcmin field of view. Spectrally the instrument samples almost the full optical domain with a mean resolution of 3000. Spatially, the instrument samples the sky with 0.2 arcseconds spatial pixels in the currently offered Wide Field Mode with natural seeing.

Closeout observations and calibrations took place during August, September and early October 2016.

MUSE recommissioning took place on 27 November 2016, with some daytime calibration, and night time data were taken on 19 and 23 November 2016.

\subsection{Image Quality}

The observations were done during a time with a seeing of 0.48 , and this resulted in a measured FWHM for the stars in the field of 0.7 and with ellipticities ranging from 0 to $0.2(1 \mathrm{~b} / \mathrm{a})$, with a median value of 0.1 . Figure 9 


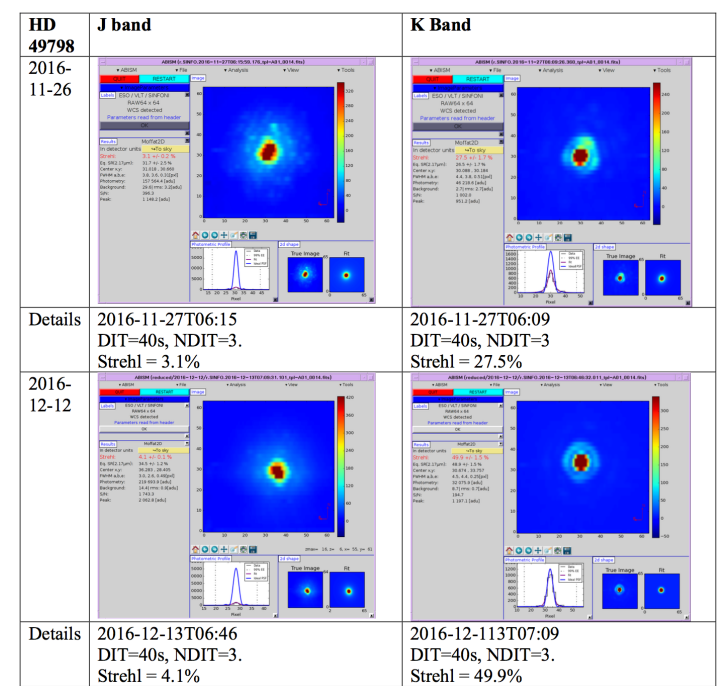

Figure 6. PSF samples for the star HD 49798 observed with SINFONI in J and K-bands. The Before and After columns refer to images obtained with the Dornier M2 unit and the new DSM M2 unit, respectively. The external seeing values were similar in both cases.

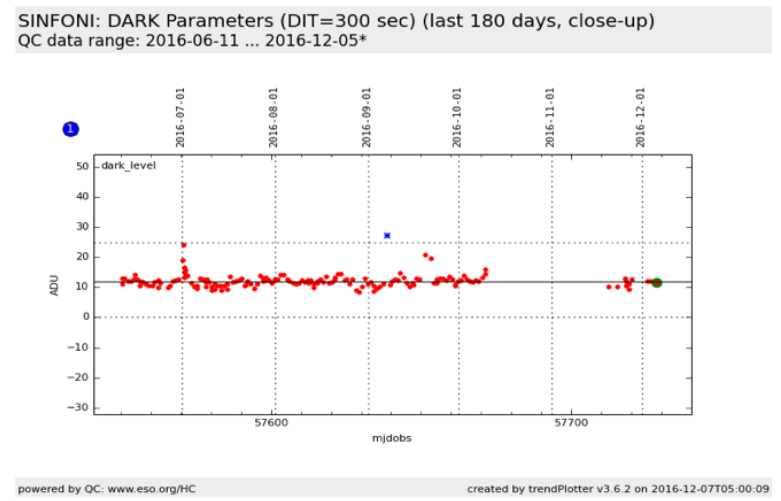

Figure 7. Evolution of the SINFONI dark level before and after the DSM instalation. The dark level was not affected by the instalation of the new DSM M2 unit.

shows the resulting histograms for the FWHM in both axis and for the ellipticity.

\subsection{Spectrophotometry}

We observed the spectrophotometric standard GD71 during closeout and commissioning. We also reduced Feige110 taken during the same closeout night, 2016-10-03, and commissioninf night 2016-11-27. To make the responses determined comparable we used the same flat fields for all reductions. These included lamp flats and twilight flats. This strategy avoid the problems that the fading blue flat lamp would have on the reductions. We did not use illumination corrections. The temperatures were approximately the same for both nights. We use the circle option instead of Moffat for the extraction of the spectrum. The Figure 10 below shows that the throughput in the blue has increased by $20-25 \%$. This has been confirmed by reducing the data with a new version of the pipeline, 1.9, that permits the comparison of standard stars observed during different nights with different flat fields.

\subsection{Astrometry}

We then defined a new astrometric field, appropriately positioned to be observable at low airmass both at the beginning of October and at the end of November. This field, NGC 1851 was chosen and show a good 


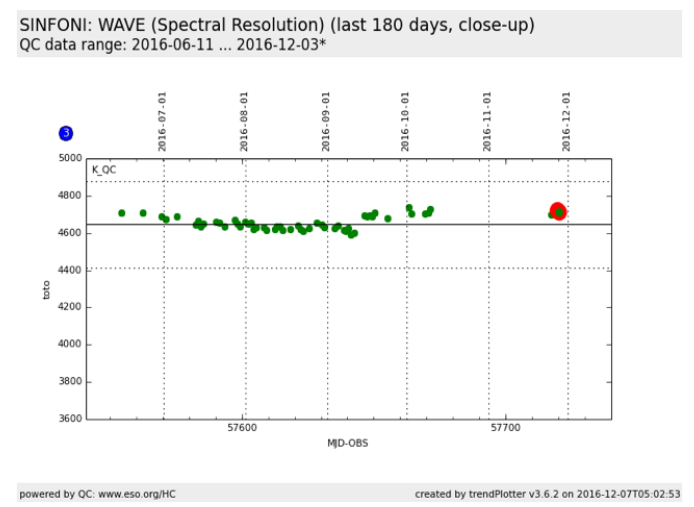

Figure 8. Evolution of the SINFONI spectral resolution before and after the DSM instalation. The spectral resolution was not affected by the instalation of the new DSM M2 unit.
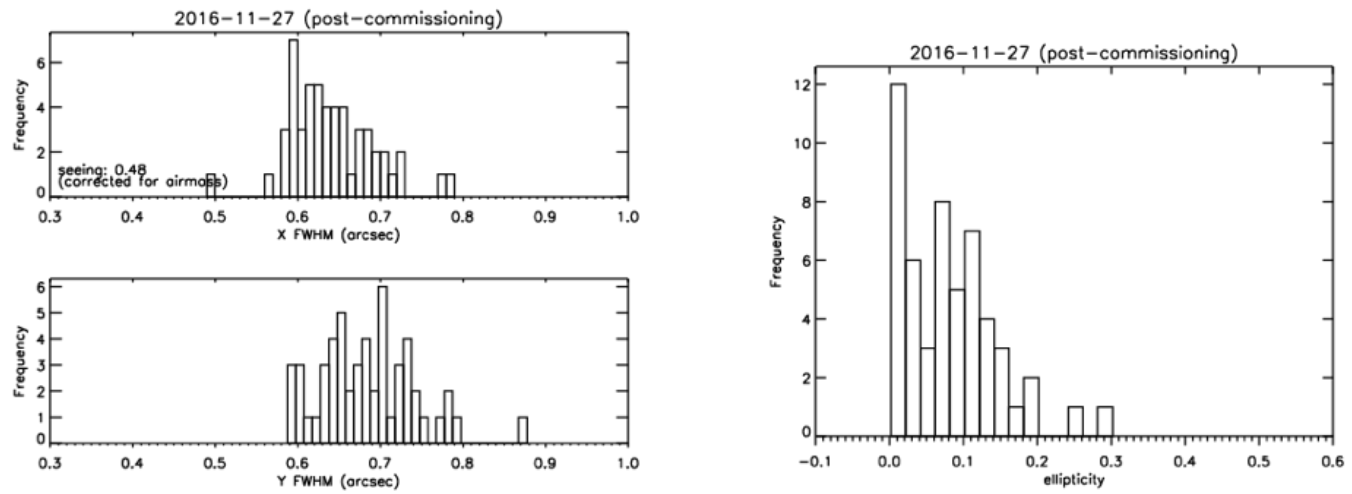

Figure 9. Histograms of the most frequent FWHM values obtained in X and Y (left figure) and of the ellipticity. Measures derived from the MUSE observations of the NGC1851 field. The median value in FWHM is located around 0.7" and the median value for the ellipticity is in the range of 0.1 .

density of star to derive a reliable comparison of the astrometric precision we obtain before and after the DSM instalation. Figure 11 presents the selected field and the difference in values between pre-commissioning and post-commissioning observations. We can confirm that the astrometry parameters are at the nominal values.

The MUSE recommissioning in November 2016 has shown that:

- All tested instrument functions behave as they should, including integration and communication with other UT4 systems.

- The Image quality is good.

- The Astrometry parameters are at nominal values

- the Spectrophotometry shows that the throughput in the blue has increased by approximately $20 \%-25 \%$.

- We were able to produce and validate an end-to-end test.

Based on these observations we can conclude that MUSE is performing well, and the DSM installation has so far not had any noticeable negative impact on performance, and perhaps an increase in throughput in the blue. 

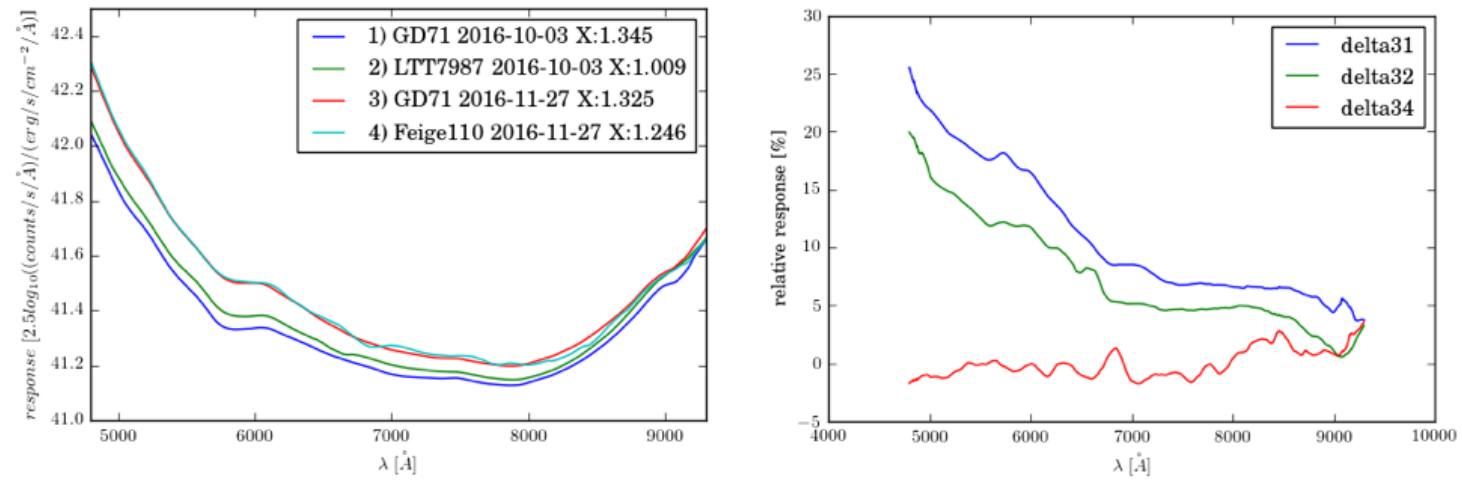

Figure 10. Derived response curves for standard stars observed during the nights of 2016-10-03 and 2016-11-27.
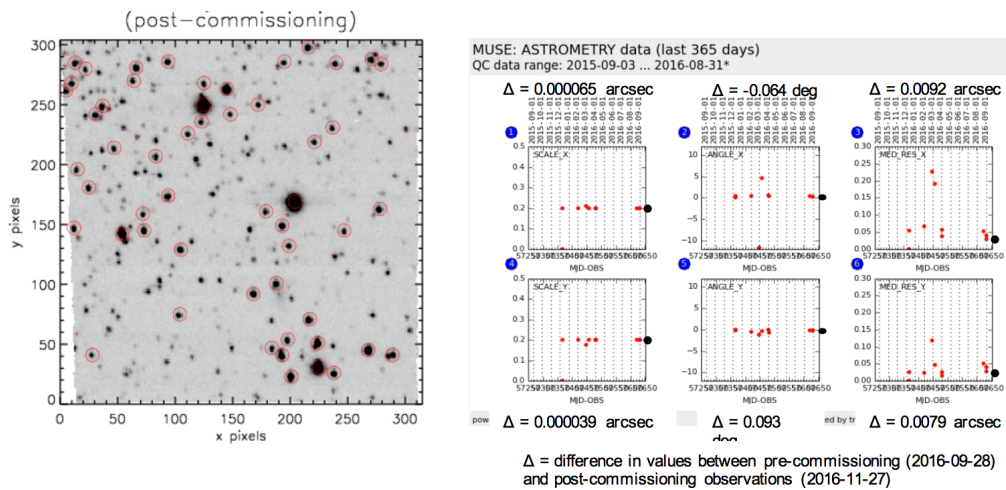

Figure 11. Observation of an astrometric field, NGC 1851, positioned to be observable at low airmass both at the beginning of October and at the end of November. The field is shown in the left figure. It has a good density of stars to allow a reliable measure of the astrometry and comparison of before and after the DSM M2 unit instalation.

\section{CONCLUSION}

From October to December 2016, UT4 was shutdown to install the new Deformable Secondary Mirror, as part of the AOF project. The excellent planning and preparation by the AOF Team allowed for a very efficient re-commissioning of the telescope and its sub-systems. Tests were also done successfully on some of the subsystems of the AOF. The excellent collaboration between the AOF Team and the team in Paranal was also a factor in the success of the various missions. Since December 19th, 5 days ahead of schedule, UT4 is back to regular operations using the new DSM in non adaptive mode. The performance of the telescope were assessed and are within the expected range. HAWK-I, MUSE and SINFONI are performing as with the original M2 and have fully resumed science operation. The VLTI instruments have also been re-commissioned with UT4 and no problem has been reported. The commissioning of the AO modules (GRAAL, GALACSI) and the DSM in AO mode have then started during the first 2017 semester.

\section{REFERENCES}

[1] Arsenault, R., Madec, P.-Y., Paufique, J., La Penna, P., Stroebele, S., Vernet, E., Pirard, J.-F., Hackenberg, W., Kuntschner, H., Kolb, J., Muller, N., Garcia-Rissmann, A., Le Louarn, M., Amico, P., Hubin, N., Lizon, J.-L., Ridings, R., Haguenauer, P., Abad, J. A., Fischer, G., Heinz, V., Kiekebusch, M., Argomedo, J., Conzelmann, R., Tordo, S., Donaldson, R., Soenke, C., Duhoux, P., Fedrigo, E., Delabre, B., Jost, A., Duchateau, M., Downing, M., Reyes Moreno, J., Manescau, A., Bonaccini Calia, D., Quattri, M., Dupuy, C., Guidolin, I. M., Comin, M., Guzman, R., Buzzoni, B., Quentin, J., Lewis, S., Jolley, P., Kraus, M., Pfrommer, T., Biasi, R., Gallieni, D., Stuik, R., Kaenders, W., Ernstberger, B., and Friedenauer, A., "ESO 
adaptive optics facility progress and first laboratory test results," in [Adaptive Optics Systems IV], 9148, 914802 (July 2014).

[2] Kolb, J., Madec, P.-Y., Arsenault, R., Oberti, S., Paufique, J., La Penna, P., Ströbele, S., Donaldson, R., Soenke, C., Suárez Valles, M., Kiekebusch, M., Argomedo, J., Le Louarn, M., Vernet, E., Haguenauer, P., Duhoux, P., Aller-Carpentier, E., Valenzuela, J. J., and Guerra, J. C., "Laboratory results of the AOF system testing," in [Adaptive Optics Systems V], 9909, 99092S (July 2016).

[3] Madec, P.-Y., Kolb, J., Oberti, S., Paufique, J., La Penna, P., Hackenberg, W., Kuntschner, H., Argomedo, J., Kiekebusch, M., Donaldson, R., Suarez, M., and Arsenault, R., "Adaptive Optics Facility: control strategy and first on-sky results of the acquisition sequence," in [Adaptive Optics Systems V], 9909, 99090Z (July 2016).

[4] Paufique, J., Madec, P.-Y., Kolb, J., Kuntschner, H., Argomedo, J., Kiekebusch, M. J., Donaldson, R. H., Arsenault, R., Siebenmorgen, R., Soenke, C., Tordo, S., Conzelmann, R. D., Jost, A., Reyes-Moreno, J., Downing, M., Hibon, P., Valenzuela, J. J., and Haguenauer, P., "GRAAL on the mountaintop," in [Adaptive Optics Systems V], 9909, 99092H (July 2016).

[5] Pirard, J.-F., Kissler-Patig, M., Moorwood, A., Biereichel, P., Delabre, B., Dorn, R., Finger, G., Gojak, D., Huster, G., Jung, Y., Koch, F., Le Louarn, M., Lizon, J.-L., Mehrgan, L., Pozna, E., Silber, A., Sokar, B., and Stegmeier, J., "HAWK-I: A new wide-field 1- to 2.5- $\mu$ m imager for the VLT," in [Ground-based Instrumentation for Astronomy], Moorwood, A. F. M. and Iye, M., eds., 5492, 1763-1772 (Sept. 2004).

[6] Casali, M., Pirard, J.-F., Kissler-Patig, M., Moorwood, A., Bedin, L., Biereichel, P., Delabre, B., Dorn, R., Finger, G., Gojak, D., Huster, G., Jung, Y., Koch, F., Lizon, J.-L., Mehrgan, L., Pozna, E., Silber, A., Sokar, B., and Stegmeier, J., "HAWK-I: the new wide-field IR imager for the VLT," in [Society of Photo-Optical Instrumentation Engineers (SPIE) Conference Series], 6269, 62690W (June 2006).

[7] Kissler-Patig, M., Pirard, J.-F., Casali, M., Moorwood, A., Ageorges, N., Alves de Oliveira, C., Baksai, P., Bedin, L. R., Bendek, E., Biereichel, P., Delabre, B., Dorn, R., Esteves, R., Finger, G., Gojak, D., Huster, G., Jung, Y., Kiekebush, M., Klein, B., Koch, F., Lizon, J.-L., Mehrgan, L., Petr-Gotzens, M., Pritchard, J., Selman, F., and Stegmeier, J., "HAWK-I: the high-acuity wide-field K-band imager for the ESO Very Large Telescope," 491, 941-950 (Dec. 2008).

[8] Siebenmorgen, R., Carraro, G., Valenti, E., Petr-Gotzens, M., Brammer, G., Garcia, E., and Casali, M., "The Science Impact of HAWK-I," The Messenger 144, 9-12 (June 2011).

[9] Eisenhauer, F., Abuter, R., Bickert, K., Biancat-Marchet, F., Bonnet, H., Brynnel, J., Conzelmann, R. D., Delabre, B., Donaldson, R., Farinato, J., Fedrigo, E., Genzel, R., Hubin, N. N., Iserlohe, C., Kasper, M. E., Kissler-Patig, M., Monnet, G. J., Roehrle, C., Schreiber, J., Stroebele, S., Tecza, M., Thatte, N. A., and Weisz, H., "SINFONI - Integral field spectroscopy at 50 milli-arcsecond resolution with the ESO VLT," in [Instrument Design and Performance for Optical/Infrared Ground-based Telescopes], Iye, M. and Moorwood, A. F. M., eds., 4841, 1548-1561 (Mar. 2003).

[10] Bacon, R., Accardo, M., Adjali, L., Anwand, H., Bauer, S., Biswas, I., Blaizot, J., Boudon, D., Brau-Nogue, S., Brinchmann, J., Caillier, P., Capoani, L., Carollo, C. M., Contini, T., Couderc, P., Daguisé, E., Deiries, S., Delabre, B., Dreizler, S., Dubois, J., Dupieux, M., Dupuy, C., Emsellem, E., Fechner, T., Fleischmann, A., François, M., Gallou, G., Gharsa, T., Glindemann, A., Gojak, D., Guiderdoni, B., Hansali, G., Hahn, T., Jarno, A., Kelz, A., Koehler, C., Kosmalski, J., Laurent, F., Le Floch, M., Lilly, S. J., Lizon, J.-L., Loupias, M., Manescau, A., Monstein, C., Nicklas, H., Olaya, J.-C., Pares, L., Pasquini, L., Pécontal-Rousset, A., Pelló, R., Petit, C., Popow, E., Reiss, R., Remillieux, A., Renault, E., Roth, M., Rupprecht, G., Serre, D., Schaye, J., Soucail, G., Steinmetz, M., Streicher, O., Stuik, R., Valentin, H., Vernet, J., Weilbacher, P., Wisotzki, L., and Yerle, N., "The MUSE second-generation VLT instrument," in [Ground-based and Airborne Instrumentation for Astronomy III], 7735, 773508 (July 2010). 